\title{
Универсальная сетевая платформа для сбора информации с датчиков и управления приборами учета
}

\author{
A. Гусаров ${ }^{1}$
}

УДК 681.58 | ВАК 05.13 .15

\begin{abstract}
Системы для умного дома, умного здания и умного города играют ключевую роль в повышении качества обслуживания, эффективности работы коммунальных служб, снижении расхода ресурсов и обеспечении безопасности городской инфраструктуры. Одно из важнейших направлений развития таких систем - автоматизация учета и контроля потребления энергоресурсов (системы АСКУЭ). Компания "Миландр" разработала эффективную платформу для АСКУЭ - гибкое сетевое решение, позволяющее не только производить учет электрической и тепловой энергии, воды, газа, но и осуществлять мониторинг, обеспечивать безопасность помещений.
\end{abstract}

дин из наиболее заметных трендов последнего десятилетия в области информационныхтехнологий - развитие Интернета вещей (ІоT), представляющего собой вычислительную сеть взаимодействующих друг с другом устройств. Данный подход позволяет радикально перестраивать технологические, экономические и общественные процессы, освобождая человека от выполнения ручных операций.

Основная задача ІоТ-систем - автоматизация взаимодействия устройств на различных уровнях, что и предопределило создание концепций умного дома, умного здания, умного города. Одно из направлений развития этих технологий - автоматизация учета и контроля потребления энергоресурсов (системы АСКУЭ).

Разработанная компанией "Миландр» сетевая платформа служит основой для организации сетевого взаимодействия в системе АСКУЭ. Главная особенность платформы - возможность работы как по выделенному проводному каналу (RS-485), так и с использованием гетерогенной сети связи на основе PLC/ RF-модулей. Модули связи могут обеспечивать обмен данными по двум интерфейсам: силовой линии напряжением 0,4 кВ при помощи PLC-технологии и радиоканалу на частоте 868 МГц. Использование существующей кабельной инфраструктуры и радиоканала позволяет избежать дополнительных затрат на прокладку кабельной сети при развертывании системы, а гетерогенность сети обеспечивает надежность работы и снижает влияние внешних условий на качество связи.

Сетевая платформа представляет собой кроссплатформенную библиотеку, позволяющую быстро интегрировать в систему различные типы устройств: от счетчиков электроэнергии до малопотребляющих датчиков с автономным питанием. В настоящее время поддерживаются платформы на базе микроконтроллера MSP430 и цифрового сигнального процессора DSP1967, разрабатывается версия для процессора ARM Cortex-M3 / M4.

Основу системы составляют выполненные в виде двух сетевых интерфейсов гетерогенные PLC/RF-модули (рис. 1), построенные на базе разработанного компанией «Миландр" сигнального процессора 1967ВН044.

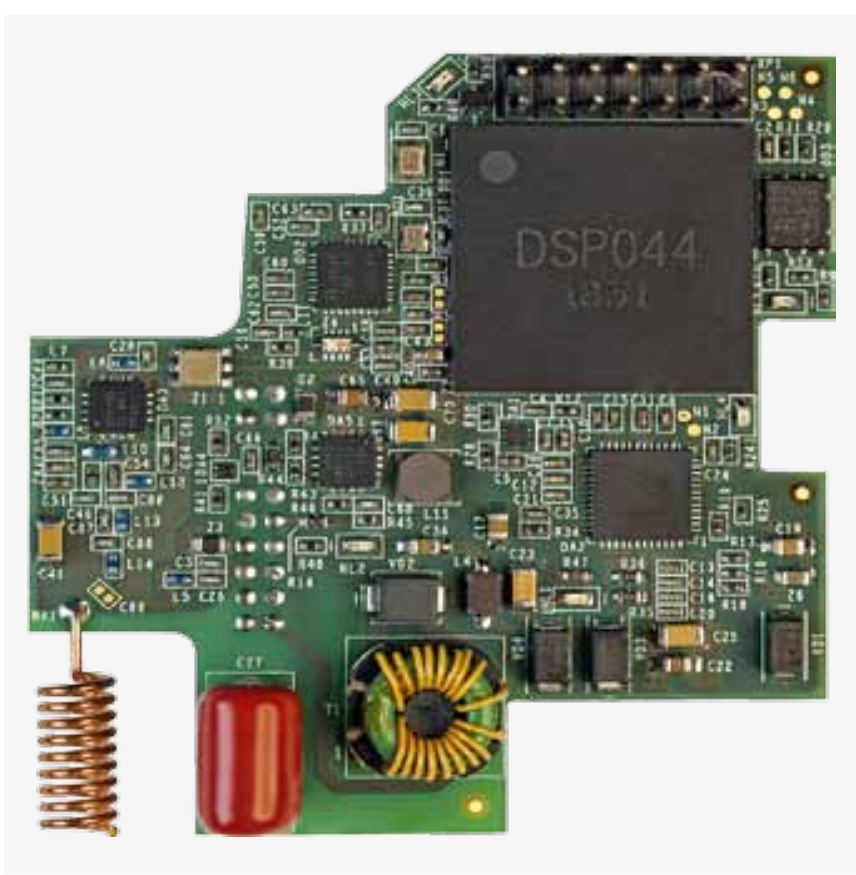

Рис. 1. Гетерогенный PLC/RF-модуль связи 


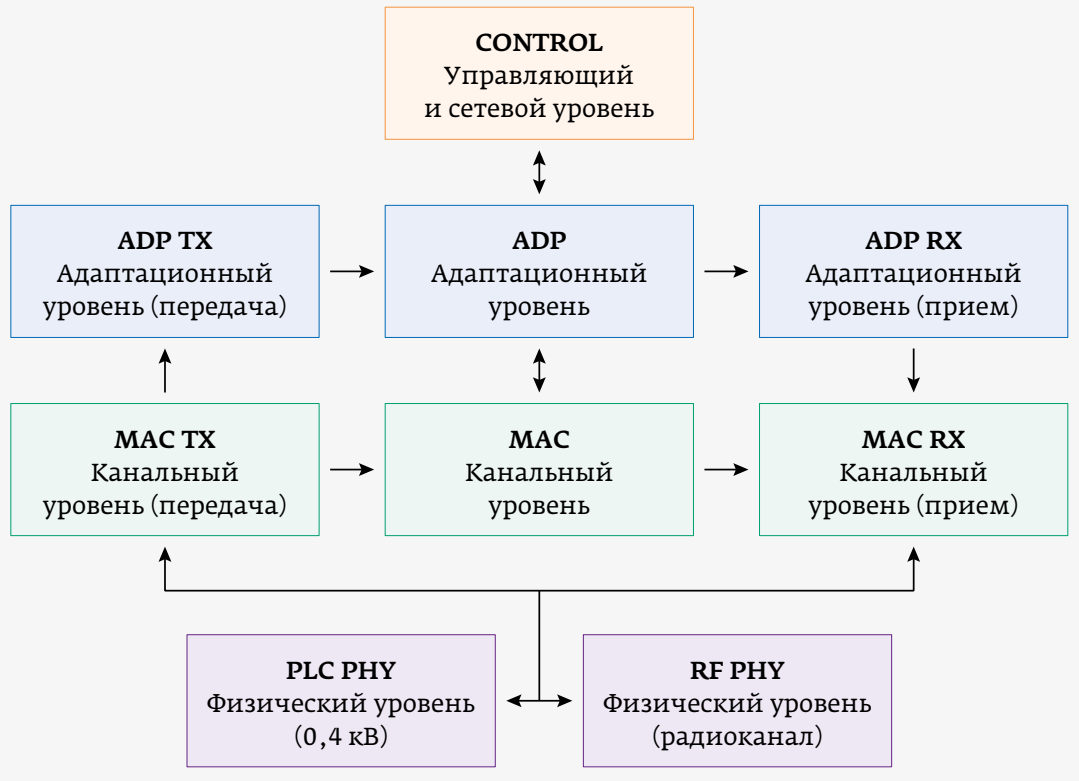

Рис. 2. Структура сетевого стека модуля связи в соответствии с последним решением ГКрч [2]. Сетевой и транспортный уровни системы обеспечивают стандартные интерфейсы IPVG и UDP для упрощения взаимодействия между устройствами (рис. 2)

Ядро системы - устройство сбора и передачи данных (УСПД), представляющее собой промышленный компьютер на основе ARM Cortex-A9. УсПД - это сетевой координатор для построения самоорганизующейся ячеистой сети приборов учета, датчиков и исполнительных механизмов [3]. Кроме того, успд обеспечивает сбор и хранение показаний всех приборов учета и взаимодействие с центральным сервером системы, предоставляющим данные для веб-интерфейса, мобильного приложения и автоматизированного рабочего места.

На рис. 3 показаны основные типы устройств системы АСКУЭ.
В устройствах с автономным питанием или при отсутствии доступа к линиям электропитания возможно применение только радиочастотного канала связи.

Физический уровень интерфейса PLC соответствует международному стандарту G3-PLC, в нем используется OFDM-модуляция для передачи данных [1]. Применение фазовой модуляции обеспечивает возможность работы даже при отрицательном соотношении сигнал/шум, а ортогональное разделение поднесущих позволяет увеличить пропускную способность канала. Использование диапазонов CENELEC A и BCD дает возможность применять систему на уровне не только поставщиков энергоресурсов, но и управляющих компаний, а также конечных потребителей. Физический уровень RF, использующий 2-GFSK-модуляцию, поддерживает режимы прослушивания канала перед началом передачи и автоматической смены каналов, что позволяет применять передатчик мощностью до 100 мВТ

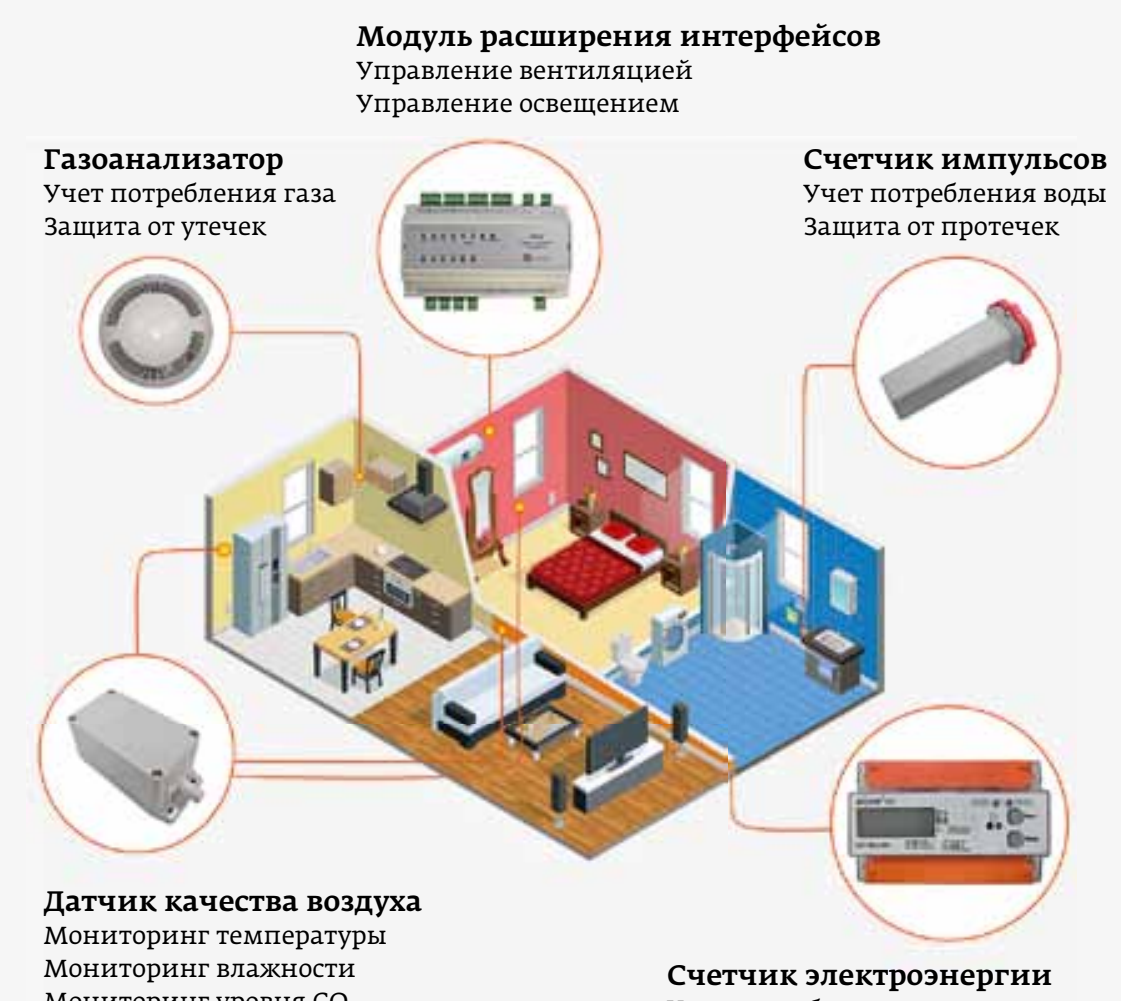

Учет потребления электроэнергии 
Для учета потребляемой электрической энергии в системе используются интеллектуальные счетчики серии "Милур". Устройства выпускаются как в трехфазном, так и в однофазном исполнении, предусматривают прямое и трансформаторное включение, что позволяет использовать их для учета электроэнергии на вводах, а также приборах конечных потребителей. С заданным временным интервалом устройства собирают и хранят срезы расхода мощности для построения профилей нагрузки и анализа потребления. Полное оснащение объекта электросчетчиками "Милур" позволяет Успд определять баланс потребления и находить утечки и неучтенные подключения. В однофазных счетчиках совместно используются шунт и токовый трансформатор для повышения качества и надежности учета. существует версия счетчиков со встроенным электромагнитным реле отключения нагрузки (80 A), которое подходит для ограничения мощности или управления уличным освещением.

Второй тип устройств в системе АСКУЭ - счетчики импульсов, которые можно использовать с приборами учета воды, тепла, газа и т. п., оснащенными импульсным выходом. Поддерживаются приборы с простым выходом типа "сухой контакт", а также устройства с выходом NAMUR для детектирования возможного обрыва или короткого замыкания импульсного провода. В связи с возможностью установки прибора в стояки, подвалы, санузлы и другие места, в которых может отсутствовать доступ к электрическим сетям напряжением 0,4кВ, он оснащен батарейным модулем, гарантирующим автономность работы до шести лет без смены источника питания [4]. Можно перевести импульсные входы в "тревожный" режим для подключения сигналов с датчиков вскрытия приборов учета, датчиков протечки и других сигналов, на которые требуется реагировать быстро.

Для объектов с подведенным газовым трубопроводом в системе используется газосигнализатор, реагирующий на природный газ или пропан-бутановую смесь. Газосигнализатор предусматривает два порога срабатывания, оснащен визуальной и звуковой сигнализацией о превышении порогов. Имеется возможность подключения внешнего газового клапана для немедленного перекрытия газа в случае аварийной ситуации. Устройство, снабженное импульсным входом, можно применять совместно со счетчиком газа с импульсным выходом для учета потребления.

Для мониторинга состояния объекта можно установить датчики микроклимата с автономным питанием. В состав прибора входят сенсоры температуры и влажности, предлагается исполнение устройства с датчиком углекислого газа. Период измерения и пороги могут настраиваться в зависимости от характеристик объекта.
Для подключения к системе устройств других производителей можно использовать модуль расширения интерфейсов, оснащенный интерфейсом RS-485, четырьмя входными и таким же количеством выходных реле. Взаимодействие с УспД возможно как по интерфейсам USB и RS-485, так и через гетерогенную PLC/ RF-сеть. Moдуль подходит для подключения альтернативных приборов учета с интерфейсом RS-485, применения в составе систем телеметрии и управления освещением и кондиционированием.

Система АСКУЭ позволяет своевременно реагировать и принимать решение при возникновении внештатных ситуаций. журналы собираемых показаний хранятся в самих приборах учета, в УспД и на центральном сервере системы. Счетчики электроэнергии поддерживают автоматическое отключение нагрузки при достижении порога превышения потребления за срез времени. В случае превышения порога загазованности на газосигнализаторе автоматически перекрывается газовый клапан вне зависимости от наличия связи с УспД и центральным сервером системы. При срабатывании электронной пломбы на счетчике импульсов все его дальнейшие показания маркируются как подозрительные, соответствующее оповещение передается на успд. Падение напряжения на батарее питания автономного датчика будет передано при следующем опросе на Успд, а затем на центральный сервер, что сформирует оповещение о необходимости ее замены. Собираемые на каждом объекте при помощи УспД данные могут быть обобщены центральным сервером на уровне нескольких объектов, а также целых микрорайонов и подстанций.

Особенность работы с сетевыми интерфейсами PLC/RF - зависимость от внешних условий среды. Радиоканал может быть заглушен, а сетевые линии подвержены помехам, для них характерно поглощение полезного сигнала. Сетевая платформа АСКУэ компании "Миландр" использует развитые алгоритмы сетевого взаимодействия приборов учета, датчиков, исполнительных механизмов и УспД для поддержания качества канала связи. Надежность передачи обеспечивается при помощи алгоритмов подтверждения и повторной отправки на канальном уровне, а проблемы с дальностью канала решаются путем ретрансляции пакетов промежуточными устройствами. Система использует алгоритм MTP (Mesh Time Protocol) для синхронизации времени между устройствами, в этом же промежутке строится остовное дерево маршрутов до координатора ячеистой сети (рис. 4).

Использование смешанной маршрутизации - проактивной от устройств к координатору и реактивной от координатора к устройствам - обеспечивает ряд преимуществ. Во-первых, сокращается время отправки сообщений о внештатных ситуациях, а во-вторых, 


\section{M МИПАнар}

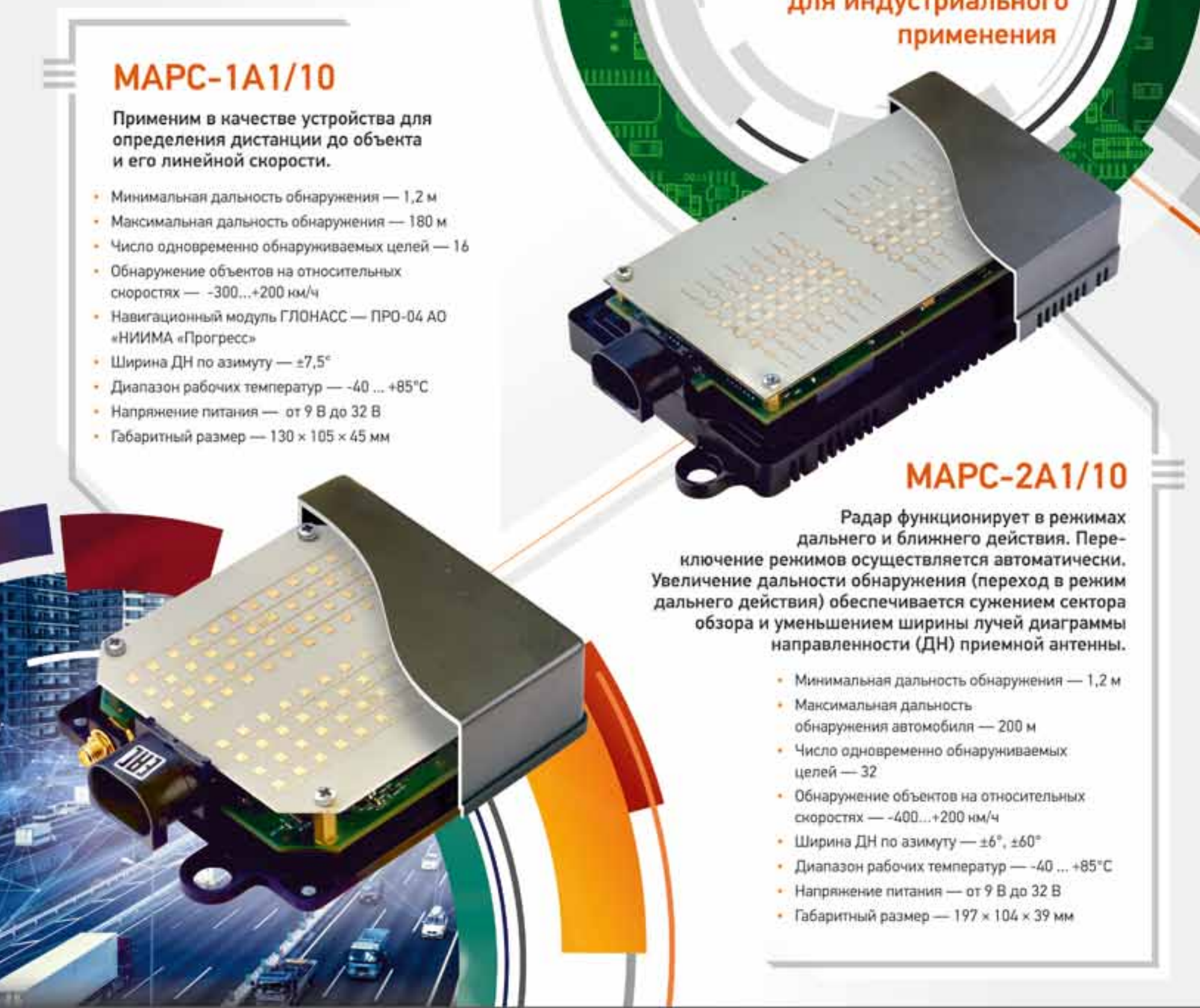

ИНДУСТРИАЛЬНЫЕ СФЕРЫ ПРИМЕНЕНИЯ СВЧ МОДУЛЕЙ

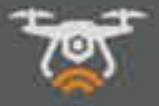

Parancвысотомеры

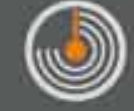

Cистемы 6esonachocm is roHiponn

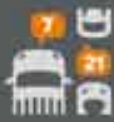

Aoporarsie cictemb $(\sqrt{5})$

Cucremei измеренit? chopocth o6 bertoe
(표)

Antomo6inehele panaper

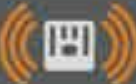

Промынunerase

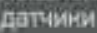

124498, г. Москва, Зеленоград, Георгиевский пр-т. А. 5

Teл.: +7 (495) 981-54-33 - Фake: +7 (495) 981-54-36

rea@milandr.ru • WWWAMILANBR RU
Техническая подяернка:

forum.milandr.ru * supportemilandr.ru

+7 (495) 221-13-55 - Wwasupportimilandr ro 


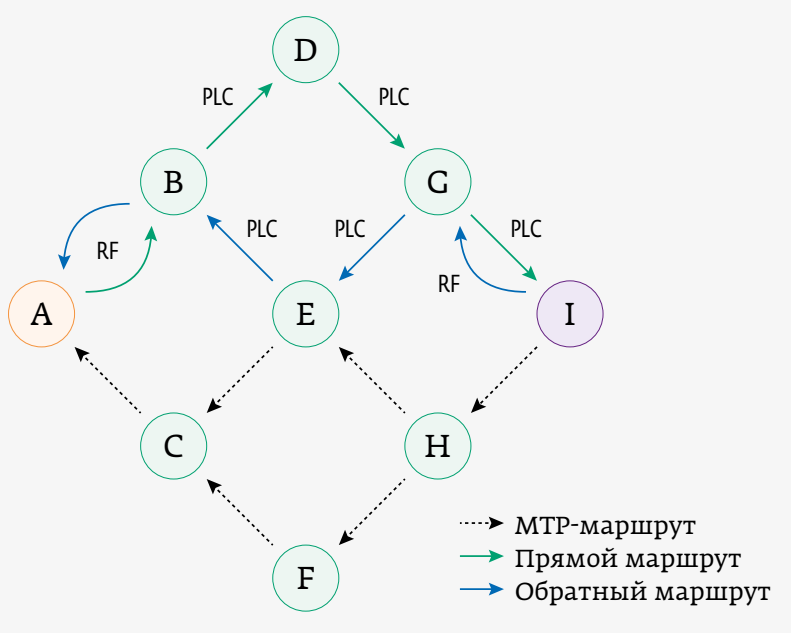

Рис. 4. Пример функционирования смешанной маршрутизации

появляется возможность гибко реагировать на изменение качества связи в каналах между устройствами [5]. При отсоединении части устройств система продолжит корректно функционировать, перестроив маршруты через оставшиеся доступные сетевые узлы. Безопасность каналов связи обеспечивается шифрованием трафика и процедурой авторизации на УспД при присоединении устройств к сети.

\section{ЛИТЕРАТУРА}

1. International Telecommunication Union -

Telecommunication sector - ITU-T G.9903 Narrowband orthogonal frequency division multiplexing power line communication transceivers for G3-PLC networks.

2. Приложение № 12 к решению ГКРЧ № 18-46-03-1 от 11 сентября 2018 года.

3. Мякочин Ю., Шедяков Д., Кареев К. УСПД на базе Cortex-А9. Вклад в построение надежных систем для ЖКХ // ЭЛЕКТРОНИКА: Наука, Технология, Бизнес. 2016. № 8

4. Мякочин Ю., Бирюков М., Гусаров А., Карпов И. Квартирный радиомодуль для системы АСКУЭ // ЭЛЕКТРОНИКА: Наука, Технология, Бизнес. 2017. № 2.

5. Гусаров А. Гетерогенная система связи, обеспечивающая когерентность устройств для построения самоорганизующихся сетей IPvG // Компоненты и технологии. 2019. № 6 .

\section{КНИГИ ИЗДАТЕЛЬСТВА «ТЕХНОСФЕРА»}

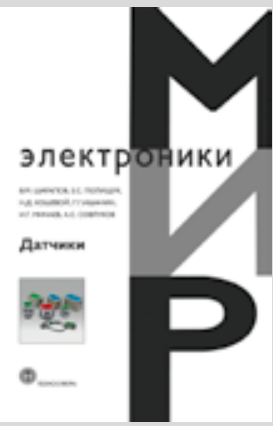

Цена 840 руб.

\section{ДАТчИКИ}

\section{В. М. Шарапов, Е. С. Полищук,} Н. Д. Кошевой, Г. Г. Ишанин, И. Г. Минаев, А.С. Совлуков

Под общ. ред. В. М. Шарапова, Е. С. Попищука

В книге изложены теоретические основы, принципы действия, описаны конструкции и характеристики датчиков физических величин. Сборник написан коллективом авторов и состоит из 24 глав. В конце каждой главы приведена обширная библиография, а также сайты предприятий-разработчиков и изготовителей.

Книга предназначена для научных работников, студентов, аспирантов, специалистов в области разработки датчиков, измерительных приборов, элементов и устройств вычислительной техники и систем управления. 
